\title{
Development of Hydrophobic Textile Materials with Organosilicon Impregnation for the Work Clothes Production
}

\author{
Liliya Yu. MAKHOTKINA \\ Ph.D. (in Technical Sciences) \\ Professor \\ Head of the Apparel and Footwear Design Department \\ Kazan National Research Technological University \\ 68, Karl Marx Str., Kazan, 420015, Russia \\ lili_makh@mail.ru \\ Alina A. KHALILOVA \\ Lecturer \\ Apparel and Footwear Design Department \\ Kazan National Research Technological University \\ 68, Karl Marx Str., Kazan, 420015, Russia \\ adikovna777@yandex.ru
}

\begin{abstract}
In recent years, special attention has been paid to studying the properties of hydrophobic surfaces of textile materials. The authors provide an overview of the applied textile-processing chemicals and of the methods for imparting hydrophobic properties to textile materials. According to the analysis, it was found that water- and splash-proofing treatment occupies an important place in the finishing processes of textile materials, contributing to a change in their characteristics. The purpose of the research is the development of hydrophobic textile materials for the work clothes production. The aqueous solution based on organosilicon compounds was used for textiles hydrophobization with retention of their performance and hygienic characteristics. The research results of the effect of aqueous solutions based on silane (A-1100 and A-187 trademarks) on the hydrophobic characteristics of textile materials is presented in the article.
\end{abstract}

Keywords: work clothes; organosilicon compounds; aqueous solution; silane (A-1100 and A-187 trademarks); hydrophobization; wetting angle; textile materials.

\section{Introduction}

Currently, the innovative development of textile and light industry is due to modern scientific discoveries and achievements of fundamental science. Innovative products include garments and textile materials with special properties. Manufacturers and consumers are particularly interested in hydrophobic materials with high hygienic characteristics; therefore, the task of textile materials hydrophobization is undoubtedly relevant.

One of the important factors in the design of high-quality work clothes is the correct selection of materials set that protects against the effects of various harmful substances and moisture. In recent years, special attention has been paid to the study of hydrophobization of textile materials surfaces (Kopachevskaya, 2016).

Today, the traditional methods of final finishing of textile materials permit to obtain hydrophobic surface with conjoined deviations in required hygienic and performance properties of the material. So, with increasing hygiene indicators, as a rule, the performance properties of the material are 
reduced. Thus, the purpose of the research is the development of hydrophobic textile materials for the work clothes production. The aqueous solution based on organosilicon compounds was used for textiles hydrophobization with retention of their operational and hygienic characteristics.

The most famous textile-processing chemicals that impart water-repellent properties to textiles include the following:

1. Emulsion forming compounds (esters, sorbitol, derivatives of hydroxycarboxylic acids, ethoxylated fatty acids, nitrogen-containing compounds, derivatives of alkylimideazoline. In this case, the hydrophobic surface is obtained by textiles soaking, after which a polymolecular layer is formed on the surface of the fiber. This layer increases hydrophobic properties due to the high adhesion to fiber (Lutfullina et. al, 2019).

2. Compounds of various chemical nature (metal complexes, compounds of aluminum salts with paraffin or wax, compounds of zirconium salts with wax, a combination of chromium and fatty acids, perfluorinated compounds of chromium and fatty acids, fatty acids, polycarboxylic acids, esters of phosphoric acid, alkyladipic acid derivatives, silicates, polyalkylhydrosiloxanes, olidimethylsiloxanes, polydimethylsiloxane rubbers, fluorocarbon resins) (Makhotkina et al., 2019; Nikolaenko, Minlebaev, 2015).

Silazanes are organosilanes that contain an amino group at the silicon atom. Amino groups are the products of the interaction of alkylchlorosilanes with ammonia. Such a compound easily comes under hydrolysis with gaseous ammonia emission, which has a harmful effect on textile fibers. Polyalkylsilazanes are used as dilute solutions in organic solvents. The fiber absorbs the solution without heat treatment (Fritzler, 2019).

Water-soluble organosilicon water-repellents, such as sodium alkyl siliconates, are widely used for hydrophobization of various materials. Due to the fact that solutions of sodium alkyl siliconates have high alkalinity, their solutions are often neutralized when used. In combination with salts of complexing metals, for example, nickel, copper, lead, zirconium, etc., sodium alkyl siliconates are used for hydrophobization of fibrous materials containing hydroxyl and ether groups (Ramamoorthy et. al., 2018).

For materials treatment, organosilicon compounds can be used in the form of water-alcohol and organic solutions or aqueous emulsions. The production of water-alcohol and organic solvents is complex and requires exhaust and recovery systems of high power. These solutions have the tendency to foam, that reduces the productivity of the equipment. Highly concentrated emulsions are more stable and can be easily diluted to the desired concentration (Makhotkina et al., 2016, Makhotkina et al., 2017).

A wide range of textile-processing chemicals is used as the final finishing to give the textile materials hydrophobic properties. In general, imparting hydrophobic properties to textile materials involves a decrease in their surface energy (Gogoi et. al. 2019). Different agents can be currently used for this purpose with varying efficiency. Agents can be arranged in a row as their hydrophobic activity increases: paraffins, silanes and siloxanes, fluorine-containing hydrocarbons (Abidi et al., 2007). For example, the authors (Shataeva, 2014) offer to use organosilicon solutions (A-1100 and A-187 trademarks) for hydrophobization of leather semi-finished products. However, these solutions have not found their application in the textile industry yet.

\section{Materials and Methods}

To study the effect of organosilicon compounds on textiles, coarse calico and severe tarp were chosen. The characteristics of the selected textile materials are shown in Table 1. As organosilicon compound silane (A-1100 and A-187 trademarks) was used.

Table 1. Characteristics of textile materials 
Fundamental and applied problems of materials creation and phases of technologies for textile industry

\begin{tabular}{cccc}
\hline Material & GOST & Article & Composition \\
\hline Coarse calico & $29298-2005$ & 262 & Cotton $100 \%$ \\
\hline Severe tarp & $15530-93$ & $11135 \mathrm{D}$ & Linen $60 \%$, \\
& & & Cotton $40 \%$ \\
\hline
\end{tabular}

At the initial stage of the research, the selection of parameters affecting the hydrophobization process was carried out. The main parameters of textile materials treatment with an aqueous solution based on organosilicon compounds are the following:

1) The concentration of silane in solution;

2) Drying temperature of the treated samples;

3) Samples soaking time in solution;

4) Impregnation solution temperature.

Using the Statistica 6.0 software package, the optimal parameters of the samples hydrophobic finish were calculated.

In this study, the following methods were used to assess changes in the surface characteristics of textile materials: the drop absorption time, the wetting angle, the stability of hydrophobic impregnation after repeated washing.

\section{1) Drop Absorption Time}

The express method was used to evaluate the hydrophobic properties of the textile material surface. The method consists in determining the drop absorption time. Testing for more than 30 minutes was considered impractical because of the drop mass loss due to evaporation (Kopachevskaya, 2016).

2) Determination of the wetting angle

DataPhysics DCAT 21 tensiometer with Wilhelmy plate measurement principle was used.

The contact angle and the wettability of solids are calculated with known surface tension and the measured force required to remove the plate from the liquid. The weight of the measured solid is balanced. A solid body is immersed in a liquid and automatically moves vertically. The values of force along the depth of immersion are captured by tensiometer and a hysteresis loop is built. The wetting angles, when immersing and raising the liquid, are calculated using the values obtained by the tensiometer (Gogoi et. al., 2019).

\section{Results and Discussion}

To obtain hydrophobic textiles the search for the optimal parameters was carried out by planning the experiment according to the following parameters: concentration of silane in the solution $(\mathrm{K})$, impregnation solution temperature $(T)$, soaking time $\left(T_{s}\right)$, drying temperature $\left(T_{d}\right)$. The determining parameter is the drop absorption time indicator $\left(\mathrm{T}_{\mathrm{da}}\right)$. Using the Statistica 6.0 software package, the optimal parameters of hydrophobic finishing of calico and tarp samples were calculated (Fig. 1-6). 

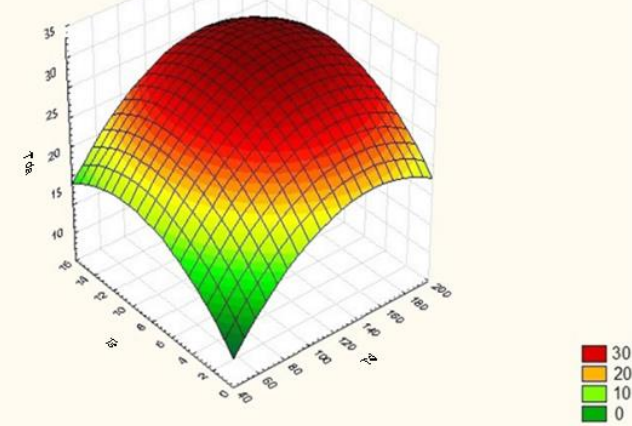

Figure 1. Optimization of the parameters of the textiles hydrophobic finishing in an aqueous solution based on silane (A-1100 tragemark). The silane concentration in solution is $5 \mathrm{~g} / \mathrm{dm}^{3}$, the impregnation solution temperature is $40^{\circ} \mathrm{C}$. The response function is the time of water drop absorption on the surface of the treated samples. Variables: drying temperature, samples soaking time in solution
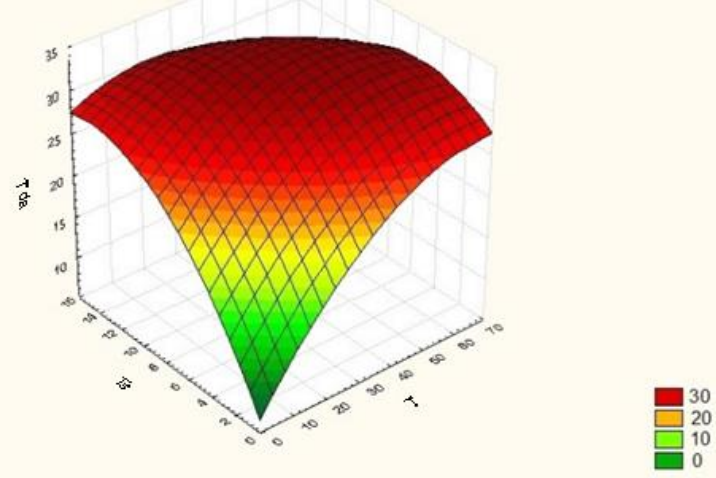

Figure 2. Optimization of the parameters of the textiles hydrophobic finishing in an aqueous solution based on silane (A-1100 tragemark). The solution concentration is 5 $\mathrm{g} / \mathrm{dm}^{3}$, the drying temperature is $160^{\circ} \mathrm{C}$. The response function is the time of water drop absorption on the surface of the treated samples. Variables: impregnation solution temperature, samples soaking time in solution

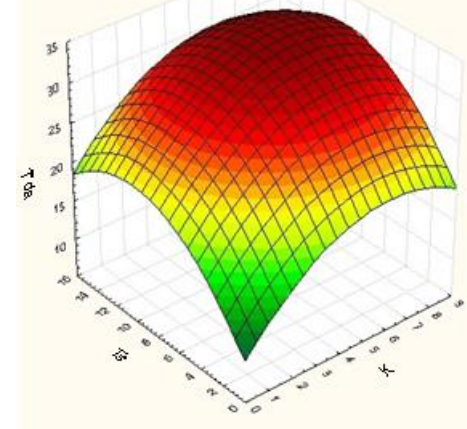

Figure 3. Optimization of the parameters of the textiles hydrophobic finishing in an aqueous solution based on silane (A-1100 tragemark). The impregnation solution temperature is $40^{\circ} \mathrm{C}$, the drying temperature is $160^{\circ} \mathrm{C}$. The response function is the time of water drop absorption on the surface of the treated samples. Variables: silane concentration in solution, samples soaking time in solution

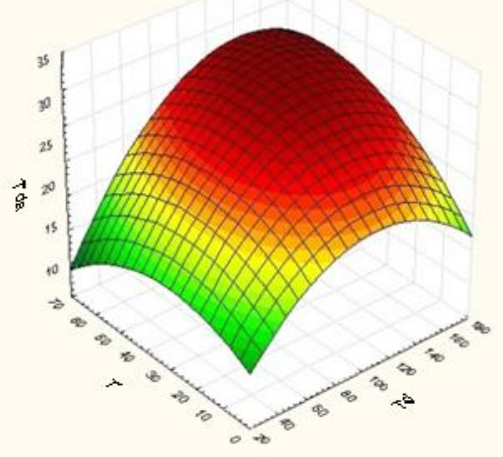

Figure 4. Optimization of the parameters of the textiles hydrophobic finishing in an aqueous solution based on silane (A-1100 tragemark). The samples soaking time in solution is 10 minutes, the drying temperature is $160^{\circ} \mathrm{C}$. The response function is the time of water drop absorption on the surface of the treated samples. Variables: silane concentration in solution, impregnation solution temperature 


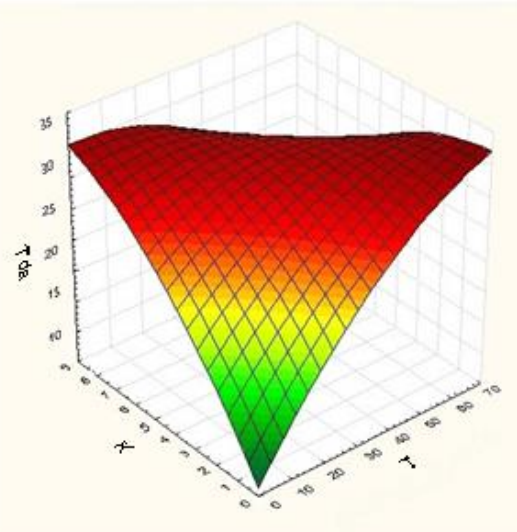

Figure 5. Optimization of the parameters of the textiles hydrophobic finishing in an aqueous solution based on silane (A-1100 tragemark). The silane concentration in solution is $5 \mathrm{~g} / \mathrm{dm}^{3}$, samples soaking time in solution is 10 minutes. The response function is the time of water drop absorption on the surface of the treated samples. Variables: drying temperature, impregnation solution temperature

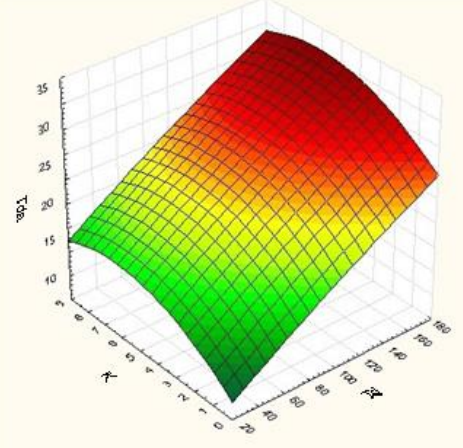

Figure 6. Optimization of the parameters of the textiles hydrophobic finishing in an aqueous solution based on silane (A-1100 tragemark). The samples soaking time in solution is $\mathbf{1 0}$ minutes, the impregnation solution temperature is $40^{\circ} \mathrm{C}$. The response function is the time of water drop absorption on the surface of the treated samples. Variables: drying temperature, silane concentration in solution

Based on the results of optimization of the four parameters of textiles hydrophobic finishing (the samples soaking time in solution, the impregnation solution temperature, the drying temperature of the samples, the silane concentration in the solution), the treatment parameters that allow obtaining hydrophobic materials were detected.

The optimal treatment parameters for textile materials to obtain hydrophobic surfaces are shown in Table 2.

Table 2. Optimal treatment parameters for textile materials to impart hydrophobic characteristics

\begin{tabular}{lcc}
\hline Treatment parameters & Coarse calico sample & Tarp sample \\
\hline Silane concentration in solution, $\mathrm{g} / \mathrm{m}^{3}$ & 5 & 5 \\
\hline Drying temperature of samples, ${ }^{0} \mathrm{C}$ & 160 & 160 \\
\hline Impregnation solution temperature, ${ }^{0} \mathrm{C}$ & 40 & 40 \\
\hline Samples soaking time in solution, min & & 10 \\
\hline
\end{tabular}

A study of the treated samples wetting angle was made to confirm the hydrophobicity of textile materials. The textiles treatment modes are presented in Table 2.

Figure 7 shows a diagram of the treated and control samples wetting angle, both for calico and tarp, each of which was impregnated by 2 types of silane (A-1100 and A-187 trademarks). 


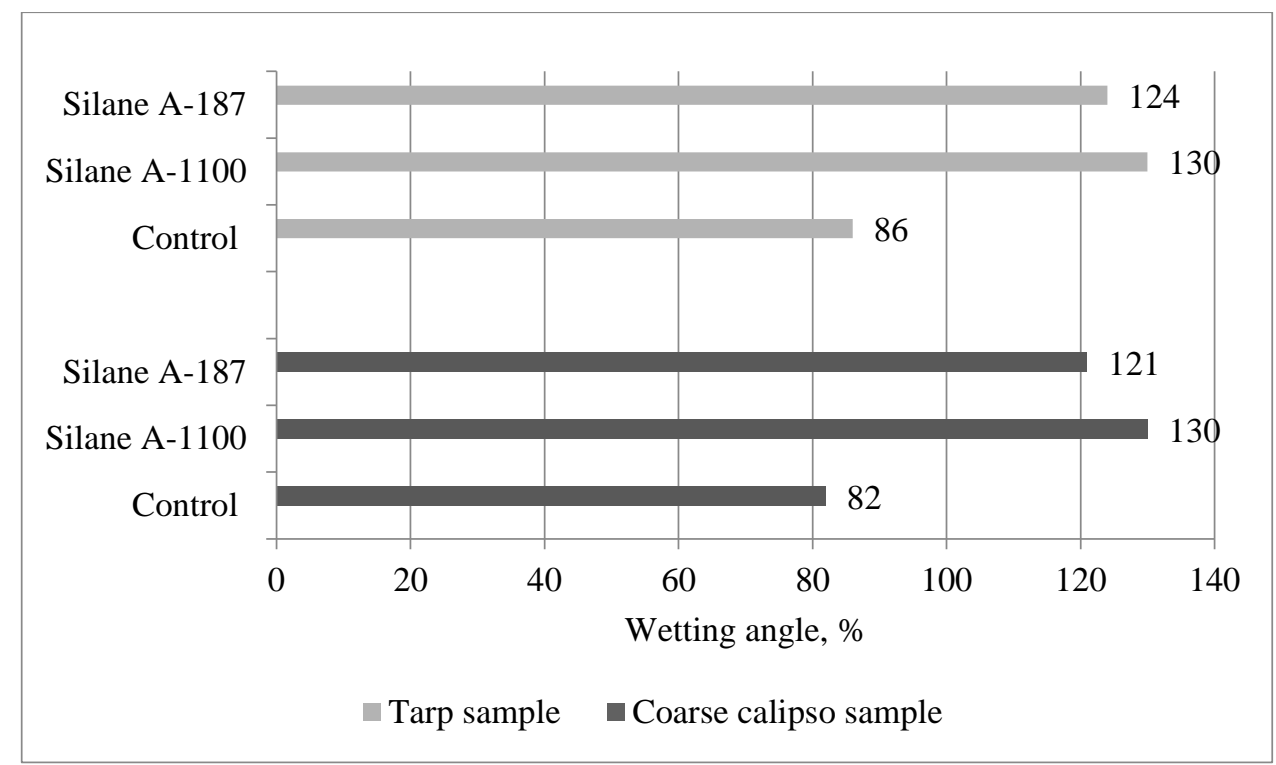

Figure 7. Values of the wetting angle of coarse calico and tarp. Silane solution concentration is $5 \mathrm{~g} / \mathrm{dm}^{3}$, impregnation solution temperature is $40^{\circ} \mathrm{C}$, samples soaking time in solution is 10 min and drying temperature is $160^{\circ} \mathrm{C}$

The measured values of the wetting angle of the calico and tarp surface confirm the results of the treatment parameters optimization using the time of water drop absorption. The results are valid both for 2 trademarks of organosilicon solutions. Thus, the hydrophobicity of textile materials increases with increasing wetting angle. For example, the value of the wetting angle of calico and tarp surfaces increases to $130^{\circ}$, after treatment with A-1100 silane solutions. The treatment with A187 silane solutions increases the measured value to $124^{\circ}$ for tarp and to $121^{\circ}$ for coarse calico.

Therefore, A-1100 silane solutions increases the hydrophobicity of materials (or the wetting angle) more intensively (by 4.6\%) as compared to A-187 silane. The wetting angle of the control samples has the essentially lower data point $(82-86 \%)$ by contrast to samples treated with silane solutions.

The multiple washing and heat treatment (ironing) were carried out to estimate the resistance of hydrophobic textiles to operational impacts of medium intensity. Stability of the hydrophobicity effect was evaluated after five washes by the drop absorption time. The experiment was carried out both for control and treated samples of calico. The results of the drop absorption time after repeated washing are shown in the Figure 8.

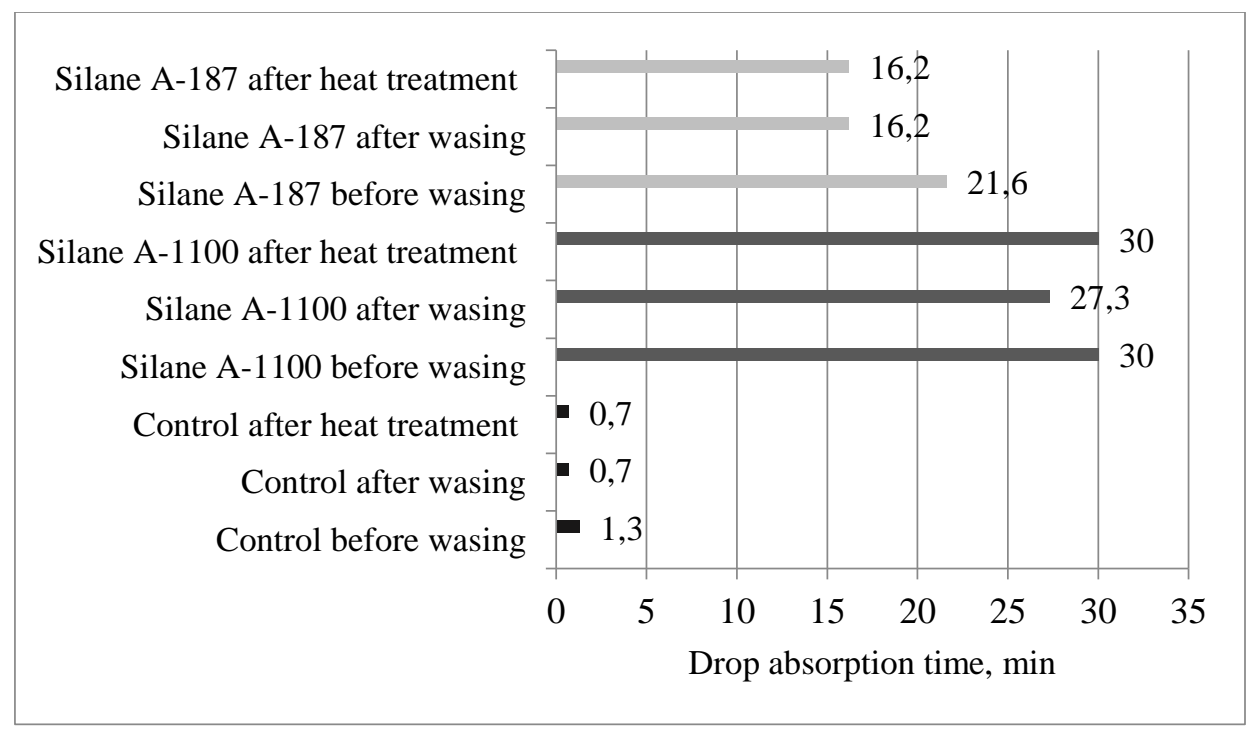

Figure 8. Drop absorption time after five washes (calico) 
As follows from Figure 8, after washing the calico control sample, the production sizing completely loses its properties and the material becomes more hydrophilic. After washing, the drop absorption time for control samples is reduced to almost 2 times and remains the same after subsequent heat treatment. Heat treatment was carried out by ironing textiles at $200^{\circ} \mathrm{C}$. Treated with silanes $(\mathrm{A}-1100$ and A-187 trademarks) hydrophobic calico loses its properties not so significantly after washing, unlike control samples. Thus, repeated washing of the treated samples reduces the drop absorption time by $10 \%$ (A-1000 silane) and by $23 \%$ (A-187 silane) relative to non-washed samples. Subsequent heat treatment does not change the drop absorption time for impregnated with A-187 silane samples. The heat treatment after washing leads to an increase in the drop absorption time of impregnated with A-1100 silane samples. In this case the drop absorption time is similar to its level for hydrophobized fabric before washing.

\section{Conclusion}

1) For the first time, the possibility of using organosilicon compounds - A-1100 and A-187 silanes as water repellents for textile materials has been shown.

2) The optimal parameters of textile impregnation were determined: silane concentration in solution - $5 \mathrm{~g} / \mathrm{dm}^{3}$; drying temperature of samples $-160^{\circ} \mathrm{C}$; impregnation solution temperature $-40^{\circ} \mathrm{C}$; samples soaking time in solution $-10 \mathrm{~min}$.

3) It was found that the processing of both calico and tarp with an aqueous solution based on silane (A-1100 and A-187 trademarks) leads to an increase in the wetting angle of about 1.5 times and to a decrease in the water drop absorption time by 30-40 times. It was determined that the hydrophobizing ability of A-1100 silane is higher than A-187 silane by $32.7 \%$ (according to drop absoption time).

4) It is shown that the hydrophobicity effect achieved by treating with silanes (A-1100 and A187 trademarks) is quite resistant to operational impacts of medium intensity, such as multiple washing and heat treatment.

\section{References}

Abidi, N., Hequet, E., Tarimala, S. (2007). Functionalization of cotton fabric with vinyltrimethoxysilane. Textile Research Journal, 77(9), 668-674.

Fritzler, A.A. (2018). Investigation of the effect of a crosslinking agent on the properties of an organosilicon coating. "Professional of the Year 2018". The XI International Research Competition - Perm: "Science and Education", 15-20. (in Russian).

Gogoi, R., Tyagi, A.K. (2019). Surface Modification of Jute Fabric by Treating with Silane Coupling Agent for Reducing Its Moisture Regain Characteristics. Journal of Natural Fibers, 18(4), 1-10.

Kopachevskaya, N.V. (2016). Development of technology for non-woven thermally bonded cloths with enhanced physical and mechanical properties. PhD thesis. Moscow. (in Russian)

Lutfullina, G.G., Makhotkina, L.Yu., Halilova, A.A. (2019). Water-repellent emulsions in the textile and light industry. Costumeology, 4(1). Retrieved from: https://kostumologiya.ru/PDF/09TLKL119.pdf (in Russian)

Makhotkina, L.Y., Khristoliubova, V.I. (2017). Studies of the influence of nonequilibrium plasma thermal exposure on the characteristics of the capillary-porous polymer material. Journal of Physics: Conference Series, 789(1), 012032.

Makhotkina, L.Y., Khristoliubova, V.I., Khannanova-Fakhrutdinova, L.R. (2016). Design of special purposes products made of nanomodified leather. IEJME: Mathematics Education, 11(6), $1495-1503$. 
Makhotkina, L.Yu., Lutfullina, G.G., Halilova, A.A. (2019). Effect of surface treatment of textile material with a solution based on organosilicon compounds on the strength characteristics of the material. "Youth and Science: a step to success". The 3rd All-Russian scientific conference of promising developments of young scientists, 281-284. (in Russian)

Nikolaenko, G.R. Minlebaev, M.N. (2015). A review of existing water-repellent materials used in light industry. Bulletin of the Technological University, 18(21), 86-87. (in Russian)

Shataeva, D.R. (2014). Development of a technology for producing a hydrophobic leather semifinished product with improved physicomechanical and hygienic properties. PhD thesis. Kazan. (in Russian) 